\title{
Shear, dilation, and swap: mixing in the limit of fast diffusion
}

\author{
Laurence Brassart ${ }^{1, a}$, Qihan Liü ${ }^{2, b}$, Zhigang Suo ${ }^{2, c}$ \\ ${ }^{1}$ Department of Materials Science and Engineering, Monash University, Clayton, \\ Victoria 3800 , Australia \\ ${ }^{2} J o h n$ A. Paulson School of Engineering and Applied Sciences, Kavli Institute for Bionano \\ Science and Technology, Harvard University, Cambridge, Massachusetts o2138, USA
}

\begin{abstract}
a) laurence.brassart@monash.edu
b) qihanliu@seas.harvard.edu

c) suo@seas.harvard.edu
\end{abstract}

\begin{abstract}
Molecules of different species mix by local rearrangement and long-range migration. Under certain conditions, the molecules are partially jammed: they rearrange slowly, but migrate fast. Here we formulate a theory of mixing when the long-range migration of molecules is fast, and the local rearrangement of molecules sets the time needed for mixing. In this limit, the time needed for mixing is independent of the length scale of inhomogeneity. We identify three modes of local rearrangement: shear, dilation, and swap. All three modes break and form intermolecular bonds. We place the three modes on equal footing, as distinct, concurrent, nonequilibrium processes. Our theory thus removes the bias that assumes local chemical equilibrium but allows the nonequilibrium process of shear. We propose a kinetic model of four independent viscosity-like coefficients, and a thermodynamic model of ideal mixing of molecules of unequal sizes and nonzero volume of mixing. We illustrate the theory with several examples, including the development of growth stress, the homogenization of a bilayer, and the disappearance of an inclusion in a matrix.
\end{abstract}

Keywords: Creep; Mixing; Reaction; Glasses; Supercooled liquids 


\section{Introduction}

In liquids and solids, molecules touch one another, but can change neighbors. This change manifests itself in experiments of two types-creep and diffusion. In creep, external forces cause a piece of material to change shape, and the resistance to the change is characterized by viscosity. In diffusion, thermal agitation causes molecules to migrate, and the rate of migration is characterized by diffusivity. In many liquids, creep and diffusion originate from the same molecular process: breaking and forming intermolecular bonds. This notion is embodied in the Stokes-Einstein relation:

$$
\frac{D \eta}{k T}=\frac{1}{C b}
$$

where $D$ is the diffusivity of a molecule migrating in a liquid, $\eta$ is the viscosity of the liquid, $k T$ is the temperature in the unit of energy, $b$ is a length characteristic of the size of the molecule, and $C$ is a dimensionless number depending on the shape of the molecule. As the temperature drops, the diffusivity decreases and the viscosity increases, but the Stokes-Einstein relation predicts that the quantity $D \eta / k T$ is a constant independent of temperature. Although Einstein (1905) derived (1) for micron-sized particles diffusing in pure liquids, the relation has been confirmed experimentally for particles as small as individual molecules diffusing in many liquids (Edward, 1970). Even in crystalline solids, the quantity $D \eta / k T$ can be temperatureindependent, provided creep and diffusion originate from the same molecular process. Examples include creep in polycrystalline solids due to self-diffusion in grains (Nabarro, 1948; Herring, 1950) or along grain boundaries (Coble, 1963). In these cases the right-hand side of (1) is replaced by a constant depending on the size of the grains. In general, a material is said to obey the Stokes-Einstein relation if $D \eta / k T$ is independent of temperature.

The Stokes-Einstein relation fails, of course, whenever creep and diffusion in a material originate from different molecular processes. For example, in a crystalline solid, when creep is 
mediated by the gliding of dislocations, and diffusion is mediated by the hopping of vacancies, the viscosity and diffusivity disobey the Stokes-Einstein relation (Frost and Ashby, 1982; Needleman and Rice, 1980). As a second example, experiments in recent decades have shown that, in some supercooled liquids, as the temperature falls toward the glass-transition point, creep slows down steeply, but diffusion does not slow down as much, so that the quantity $D \eta / k T$ increases by orders of magnitude (Angell et al., 2000; Debenedetti and Stillinger, 2001; Faupel et al., 2003; Ediger and Harrowell, 2012). Such a supercooled liquid may form a dynamically heterogeneous structure that nearly jams creep, but still allows diffusion (Berthier 2011; Ediger and Harrowell, 2012). As a third example, in a physical gel, polymer chains crosslink by physical bonds that reversibly break and form, and the molecules of the solvent migrate through the network of polymers. Under certain conditions, the time needed for the mixing of the polymers and the solvent is set by the breaking and forming of the physical crosslinks, not by the migration of solvent (Zhao et al., 2010; Hu and Suo 2012).

Whereas the microscopic mechanisms that break the Stokes-Einstein relation are material-specific, macroscopic behavior can be described, independent of microscopic mechanisms, by a continuum theory that regards creep and diffusion as distinct processes. Analogous to poroelasticity that studies concurrent diffusion and elastic deformation, poroviscosity studies concurrent diffusion and creep. We have formulated a poroviscous theory of concurrent self-diffusion and creep in pure substances (Suo, 2004; Pharr et al., 2011; Li et al., 2014). Here we turn to mixtures of molecules of different species. Mixtures often separate into phases, but this paper will focus on solutions of single phases.

Consider a solution of two species of molecules. The binary solution is initially inhomogeneous over some length $L$, and evolves toward a homogeneous state. The time needed to homogenize the solution by diffusion scales as $\tau_{D} \sim L^{2} / D$. The time needed to homogenize the solution by creep scales as $\tau_{C} \sim \eta \Omega / k T$, where $\Omega$ is the mean volume per molecule (Section 
2.5). When $\tau_{D}<<\tau_{C}$, the rate of mixing is limited not by diffusion, but by creep. Thus, creeplimited mixing prevails over diffusion-limited mixing when

$$
L \ll \sqrt{\frac{D \eta \Omega}{k T}} .
$$

The quantity on right side is a material-specific length, $\sqrt{D \eta \Omega / k T}$, which we call the poroviscous length. For a material that violates the Stokes-Einstein relation, the poroviscous length changes with temperature, and can be much larger than the size of individual molecules. The poroviscous length has also appeared in the theory of concurrent creep and self-diffusion in pure substances (Suo, 2004; Pharr et al., 2011; Li et al., 2014), as well as in a theory of Brownian motion of molecular probes in supercooled liquids (Liu et al., 2015).

As noted above, when the length scale of inhomogeneity in a solution is small compared to the poroviscous length, the time needed for mixing is set by creep, not by diffusion. This paper devotes to viscous mixing in the limit of fast diffusion. In this limit, the time needed for mixing is independent of the length scale of inhomogeneity. To focus on viscous mixing, we neglect elasticity, and assume that diffusion is so fast that chemical potentials of the two species of molecules in the solution are uniform. We will discuss viscoelastic mixing and poroviscous mixing elsewhere. Viscous mixing studied here differs greatly from that commonly studied in hydrodynamics, where the Stokes-Einstein relation holds, molecules diffuse slowly, and stirring accelerates mixing.

1.1 Link with theories coupling mechanics and diffusion

Our work is directly relevant to a number of problems in materials that couple inelastic deformations and diffusion. Continuum theories have been proposed for a large number of systems, including crystals (Svoboda et al., 2006; Mishin et al., 2013), glasses (Stephenson, 1988), oxides (Suo et al., 2003; Loeffel and Anand, 2011), polymers (Govindjee and Simo, 1993), 
and soils and partially molten rocks (McKenzie, 1984; Viesca et al., 2008). The study of concurrent inelastic deformations and species interdiffusion in solids has recently gained increased interest with the fast development of novel energy-storage materials such as highcapacity electrodes for lithium-ion batteries (Bower et al., 2011, Cui et al. 2012, Brassart et al., 2013; Drozdov 2014; Di Leo et al., 2015). Another growing field to which our work is closely related addresses the coupling between large, possibly inelastic deformations and diffusion in soft materials such as hydrogels (Zhao et al., 2010; Chester 2012; Hu and Suo, 2012; Wang and Hong, 2012; Strange et al., 2013).

Most of the existing formulations - including the ones listed above- rely on the assumption of local chemical equilibrium. The concentration of a mobile species at a given macroscopic point in one-to-one related to the chemical potential by a state law. The long-range transport of species is then the only rate-limiting process. This corresponds to the approach of the classical thermodynamics of irreversible processes (de Groot and Mazur). In contrast, this approach allows the local non-equilibrium process of shear deformations by creep. The fundamentally different treatment of creep and species insertion does not seem justified when both processes are mediated by similar molecular rearrangements. Such a theory cannot describe viscous mixing in the limit of fast diffusion.

Brassart and Suo $(2012,2013)$ recently formulated a theory that regards both flow and species insertion as non-equilibrium processes, placing their respective driving forces on the same footing. This so-called "Reactive Flow" theory was motivated by experimental observations of low yield stress of amorphous silicon during the lithiation. The paper focused on the formulation of coupled chemical-mechanical plasticity models in host-guest systems (one diffusing species), and also included elasticity. The idea of non-equilibrium insertion was also included in our recent paper on concurrent self-diffusion and creep in pure substances (Li et al., 2014), but its consequences on the predictions were not studied in details. Here we provide a comprehensive treatment of this idea for binary mixtures. 
By relaxing the assumption of local chemical equilibrium, our formulation is related to theories of case II diffusion in polymers (see Bargmann et al. (2011) for a recent review). One approach to case II diffusion consists in including a dependence on pressure in the expression of the chemical potential. Insertion of species is then limited by pressure relaxation due to viscoelasticity (Thomas and Windle, 1982; Durning, 1985). In contrast, our theory predicts ratelimited insertion even in the absence of stress.

\subsection{Mechanisms of viscous mixing}

Imagine a small piece of solution, subject to a state of stress, in chemical contact of reservoirs of the two species of molecules. During viscous mixing, the solution rearranges molecules by shear, dilation, and swap (Fig. 1). The piece shears when it conserves the numbers of the two species of molecules, but changes shape. The piece dilates when it preserves the ratio of the two species of molecules and the shape, but changes the volume. The piece swaps when it preserves the shape and the volume, but changes the ratio of the two species of molecules. Diffusion is so fast that the reservoirs rapidly broadcast molecules to the solution, and the chemical potentials of the molecules in the solution are the same as those in the reservoirs. Consequently, during dilation and swap, the two species of molecules mix in the interior of the solution. 


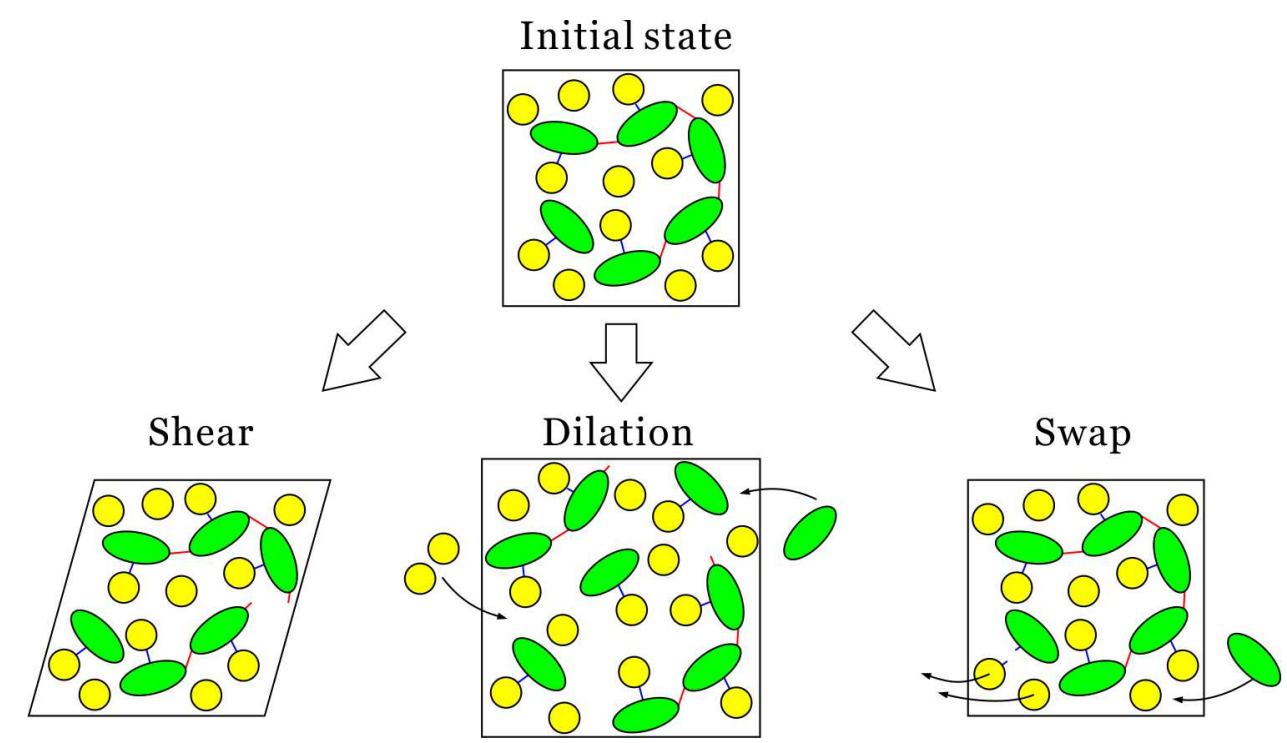

Fig. 1. A piece of a binary solution evolves in three modes. Shear: the piece preserves the number of each species of molecules, but changes the shape. Dilation: the piece preserves the ratio of the two species of molecules and the shape, but changes the volume. Swap: the piece preserves the shape and the volume, but changes the ratio of the two species of molecules.

All three modes of molecular rearrangement-shear, dilation, and swap-break and form intermolecular bonds. We place the three modes on equal footing, as distinct, concurrent, nonequilibrium processes. Our theory thus removes the bias that assumes local chemical equilibrium but allows the nonequilibrium process of shear. This bias is untenable when local rearrangement of molecules limits the rate of mixing, even though it is commonly held in theories of concurrent creep and diffusion; see papers cited above.

We propose a kinetic model of four independent viscosity-like coefficients, and consider ways to determine them experimentally. We also derive a thermodynamic model of ideal mixing of molecules of unequal sizes and nonzero volume of mixing. We use the theory to analyze steady, transient, and inhomogeneous viscous mixing. Examples illustrate the development of growth stress, the homogenization of a bilayer, and the disappearance of an inclusion in a matrix.

\section{Theory of homogeneous viscous mixing}


Consider a binary solution of two species of molecules A and B, subject to mechanical forces, in chemical contact with reservoirs of the two species of molecules (Fig. 2). The reservoirs may contain molecules other than species $\mathrm{A}$ and $\mathrm{B}$, but the chemical contact is permeable only to species $\mathrm{A}$ and $\mathrm{B}$, so that the piece of the solution always consists of the two species only. For the time being, we assume that the piece of the solution is large enough to behave like a representative volume element, but is small enough to evolve through a sequence of homogeneous states.

\subsection{Kinematics}

We specify the kinematics of homogeneous viscous mixing by rates of deformation and injection. Denote the rate of deformation by a symmetric tensor as a function of time, $d_{i j}(t)$. Let the volume of the solution be $V(t)$. The trace of the rate of deformation equals the rate of dilation, $d_{k k}=\dot{V} / V$. A superimposed dot indicates a derivative with respect to time. Denote the deviatoric part of the rate of deformation by $e_{i j}=d_{i j}-d_{k k} \delta_{i j} / 3$. The symmetric and traceless tensor $e_{i j}$ have five independent components, and describe the rate of shear, namely, the rate at which the solution changes its shape.

The piece of the solution contains $n_{A}(t)$ molecules of species A and $n_{B}(t)$ molecules of species B. We specify the composition of the solution by the number fraction of one of the species, $\xi=n_{B} /\left(n_{A}+n_{B}\right)$. The composition, in general, also changes with time, $\xi(t)$. The reservoirs inject the two species of molecules into the solution at the rates of $\dot{n}_{A}$ and $\dot{n}_{B}$. Analogous to the rate of deformation, which is independent of the size of the piece of the solution, the rates of injection may be defined by $\dot{n}_{A} / V$ and $\dot{n}_{B} / V$, or by $\dot{n}_{A} /\left(n_{A}+n_{B}\right)$ and $\dot{n}_{B} /\left(n_{A}+n_{B}\right)$. We will define another measure of the rates of injection below. 


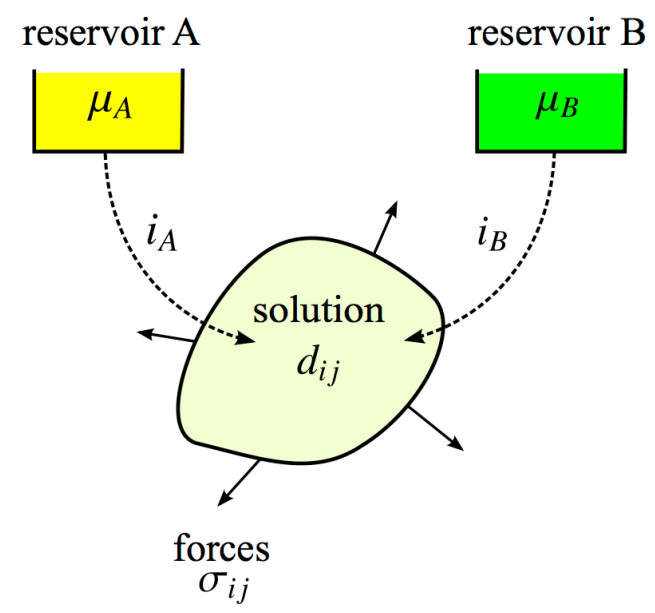

Fig. 2. A piece of binary solution is in chemical contact with reservoirs of species A and B, and is subject to external forces. The piece evolves by the rates of injection and the rate of deformation.

\subsection{Incompressibility}

For a solution of fixed numbers of the two species of molecules, the volume of the solution is taken to be independent of the applied stress. This assumption of molecular incompressibility is accurate so long as the applied stress is small compared to the intermolecular interaction. Under this assumption, the volume of the solution is a function of the numbers of molecules of the two species, $V\left(n_{A}, n_{B}\right)$.

The partial derivatives, $V_{A}=\partial V\left(n_{A}, n_{B}\right) / \partial n_{A}$ and $V_{B}=\partial V\left(n_{A}, n_{B}\right) / \partial n_{B}$, are known as the partial volumes of the two species of molecules in the solution. Because the volume of the solution is an extensive variable, $V\left(n_{A}, n_{B}\right)$ is a homogeneous function of degree one, and the partial volumes are functions of composition, $V_{A}(\xi)$ and $V_{B}(\xi)$. At a given time, the volume of the solution is $V=V_{A}(\xi) n_{A}(t)+V_{B}(\xi) n_{B}(t)$, and changes at the rate $\dot{V}=V_{A}(\xi) \dot{n}_{A}+V_{B}(\xi) \dot{n}_{B}$. 
We call $i_{A}=V_{A} \dot{n}_{A} / V$ and $i_{B}=V_{B} \dot{n}_{B} / V$ the rates of (volumetric) injection of the two species. These two rates are also independent of the size of the piece of the solution. A direct calculation gives that

$$
\dot{\xi}=\frac{(1-\xi) \Omega(\xi) i_{B}}{V_{B}(\xi)}-\frac{\xi \Omega(\xi) i_{A}}{V_{A}(\xi)} .
$$

Here $\Omega$ is the mean volume of the solution, $\Omega=V /\left(n_{A}+n_{B}\right)=(1-\xi) V_{A}(\xi)+\xi V_{B}(\xi)$. Recall that the rate of dilation is $\dot{V} / V=d_{k k}$, so that

$$
d_{k k}=i_{A}+i_{B} .
$$

The assumption of molecular incompressibility thus relates the rate of dilation to the rates of volumetric injection of the two species.

The deviatoric part of the rate of deformation, along with the rates of injection, constitutes a total of seven independent rates, $\left(i_{A}, i_{B}, e_{i j}\right)$. Equivalently, we can specify the rate of shear by $e_{i j}$, the rate of dilation by $d_{k k}$, and the rate of swap by $\dot{\xi}$. For a solution of a given composition, (3) and (4) constitute a linear map between $\left(i_{A}, i_{B}\right)$ and $\left(\dot{\xi}, d_{k k}\right)$. Inverting the linear map, we obtain that

$$
\begin{gathered}
i_{A}=-\frac{V_{A} V_{B}}{\Omega^{2}} \dot{\xi}+\frac{(1-\xi) V_{A}}{\Omega} d_{k k}, \\
i_{B}=\frac{V_{A} V_{B}}{\Omega^{2}} \dot{\xi}+\frac{\xi V_{B}}{\Omega} d_{k k} .
\end{gathered}
$$

These equations express the rates of injection, $\left(i_{A}, i_{B}\right)$, in terms of the rates of swap and dilation, $\left(\dot{\xi}, d_{k k}\right)$. We regard $\left(i_{A}, i_{B}, e_{i j}\right)$ and $\left(\dot{\xi}, d_{k k}, e_{i j}\right)$ as alternative representations of the same kinematic state. 


\subsection{Thermodynamics}

We now associate the seven kinematic rates with seven thermodynamic forces. The external forces, the reservoirs, and the solution together constitute a composite thermodynamic system. We only consider isothermal processes, and do not list temperature as a variable. The external forces acting on the solution result in a state of stress $\sigma_{i j}$. As the solution deforms, the potential energy of the mechanical forces changes at the rate of $-\sigma_{i j} d_{i j} V$. The reservoirs have chemical potentials of the two species of molecules, $\mu_{A}$ and $\mu_{B}$. As the reservoirs inject molecules into the solution, the free energy of the reservoirs changes at the rate $-\mu_{A} \dot{n}_{A}-\mu_{B} \dot{n}_{B}$.

We assume that the Helmholtz free energy of the solution is also independent of the applied stress - that is, we neglect the elasticity of the solution. The Helmholtz free energy of the solution is a function of the numbers of molecules of the two species, $F\left(n_{A}, n_{B}\right)$. Define the partial Helmholtz free energies by $F_{A}=\partial F\left(n_{A}, n_{B}\right) / \partial n_{A}$ and $F_{B}=\partial F\left(n_{A}, n_{B}\right) / \partial n_{B}$. Because the Helmholtz free energy of the solution is an extensive variable, $F\left(n_{A}, n_{B}\right)$ is a homogeneous function of degree one, and the partial free energies of the solution are functions of its composition, $F_{A}(\xi)$ and $F_{B}(\xi)$. At a given time, the free energy is $F=F_{A}(\xi) n_{A}+F_{B}(\xi) n_{B}$, and changes at the rate $\dot{F}=F_{A}(\xi) \dot{n}_{A}+F_{B}(\xi) \dot{n}_{B}$.

In general, the composite system is not in thermodynamic equilibrium. The Helmholtz free energy of the composite system is the sum over the parts (i.e., the solution, the reservoirs, and the external forces). The composite system interacts with the surroundings through the transfer of energy by heat only. Consequently, thermodynamics requires that the Helmholtz free energy of the composite system should never increase:

$$
\left(F_{A} \dot{n}_{A}+F_{B} \dot{n}_{B}\right)-\left(\mu_{A} \dot{n}_{A}+\mu_{B} \dot{n}_{B}\right)-V \sigma_{i j} d_{i j} \leq 0 .
$$


The equality holds when the composite system is in a state of thermodynamic equilibrium, and the inequality holds when the composite system evolves.

Define the mean stress by $\sigma_{m}=\sigma_{k k} / 3$, and the deviatoric stress by $s_{i j}=\sigma_{i j}-\sigma_{m} \delta_{i j}$. Note the identity $\sigma_{i j} d_{i j}=s_{i j} e_{i j}+\sigma_{m} d_{k k}$. Using $i_{A}=V_{A} \dot{n}_{A} / V, i_{B}=V_{B} \dot{n}_{B} / V$, and $d_{k k}=i_{A}+i_{B}$, we rewrite the above thermodynamic inequality as

$$
\left(\frac{\mu_{A}-F_{A}}{V_{A}}+\sigma_{m}\right) i_{A}+\left(\frac{\mu_{B}-F_{B}}{V_{B}}+\sigma_{m}\right) i_{B}+s_{i j} e_{i j} \geq 0 .
$$

We have expressed this thermodynamic inequality in terms of the seven independent rates $\left(i_{A}, i_{B}, e_{i j}\right)$

Inequality (8) identifies $\zeta_{A} \equiv\left(\mu_{A}-F_{A}\right) / V_{A}+\sigma_{m}$ and $\zeta_{B} \equiv\left(\mu_{B}-F_{B}\right) / V_{B}+\sigma_{m}$ as the thermodynamic forces conjugate to the rates of volumetric injection $i_{A}$ and $i_{B}$. These chemical driving forces represent the imbalance between the chemical potentials in the reservoir and the partial free energies in the solution. Due to the assumption of molecular incompressibility, the solution changes volume on receiving molecules, so that the mean stress contributes to the two chemical driving forces. Inequality (8) also identifies the deviatoric stress $s_{i j}$ as the mechanical driving force conjugate to the deviatoric rate of deformation $e_{i j}$. The deviatoric stress $s_{i j}$ is a symmetric and traceless tensor, and represents a total of five independent mechanical driving forces. In total, inequality (8) identifies seven thermodynamic forces $\left(\zeta_{A}, \zeta_{B}, s_{i j}\right)$ conjugate to the seven independent rates $\left(i_{A}, i_{B}, e_{i j}\right)$. In the language of linear algebra, the seven rates are the components of a vector, and the seven thermodynamic forces are the components of another vector. The two seven-dimensional vector spaces are dual spaces with respect to a scalar setthe rate of change in free energy per unit volume. 
We can also express the thermodynamic inequality in terms of the rates of shear, dilation, and swap, $\left(e_{i j}, d_{k k}, \dot{\xi}\right)$. Inserting (5) and (6) into (8), we obtain that

$$
\frac{\left(\mu_{B}-F_{B}\right) V_{A}-\left(\mu_{A}-F_{A}\right) V_{B}}{\Omega^{2}} \dot{\xi}+\left(\frac{\left(\mu_{A}-F_{A}\right)(1-\xi)+\left(\mu_{B}-F_{B}\right) \xi}{\Omega}+\sigma_{m}\right) d_{k k}+s_{i j} e_{i j} \geq 0
$$

This inequality identifies the thermodynamic forces conjugate to the rate of swap $\dot{\xi}$ and the rate of dilation $d_{k k}$.

\subsection{Equilibrium}

The composite system is in a state of thermodynamic equilibrium when the equality in (8) holds. That is, thermodynamic equilibrium requires that all seven thermodynamic forces vanish, so that

$$
\begin{gathered}
s_{i j}=\mathrm{O}, \\
F_{A}(\xi)-\sigma_{m} V_{A}(\xi)=\mu_{A}, \\
F_{B}(\xi)-\sigma_{m} V_{B}(\xi)=\mu_{B} .
\end{gathered}
$$

These conditions of thermodynamic equilibrium correspond to a total of seven independent equations: $\sigma_{12}=\sigma_{23}=\sigma_{31}=0$ and $\sigma_{11}=\sigma_{22}=\sigma_{33}=\left(F_{A}-\mu_{A}\right) / V_{A}=\left(F_{B}-\mu_{B}\right) / V_{B}$. For the binary solution, mechanical forces, and chemical reservoir to equilibrate, the solution must be in a hydrostatic state of stress, and the hydrostatic stress must balance the difference between the chemical potentials of the two species in the reservoir and the partial free energies of the two species in the solution.

Equations (11) and (12) recover the well-known conditions of thermodynamic equilibrium: the partial Gibbs function for each species is the same in all parts in chemical contact. The two equations of equilibrium relate four variables: the composition of the solution 
$\xi$, the hydrostatic stress acting on the solution $\sigma_{m}$, and the chemical potentials of the two species of molecules in the reservoirs, $\mu_{A}$ and $\mu_{B}$. For example, when the chemical potentials of the two species of molecules in the reservoirs are fixed at known values, $\mu_{A}$ and $\mu_{B}$, both the hydrostatic stress $\sigma_{m}$ acting on the solution and the composition $\xi$ of the solution must adjust for the composite system to reach a state of thermodynamic equilibrium. The hydrostatic stress that equilibrates the solution and the reservoir is known as the osmotic stress (Fig. 3).

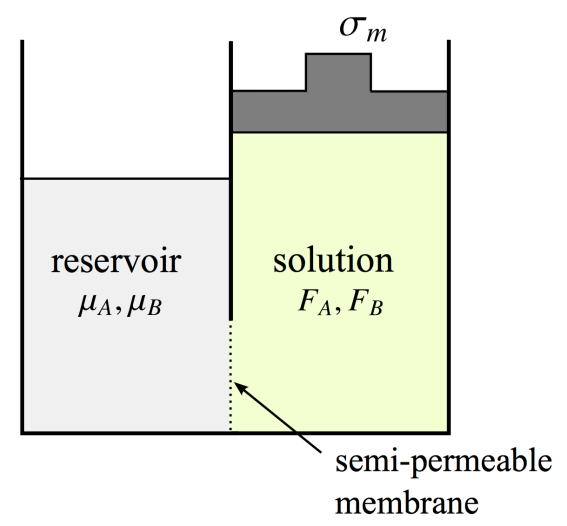

Fig. 3. A binary solution is in chemical contact with a reservoir of the two species of molecules, and is subject to a hydrostatic stress. The hydrostatic stress needed for the composite system to reach thermodynamic equilibrium is called the osmotic stress.

\subsection{Kinetics}

The composite system is out of thermodynamic equilibrium when the inequality in (8) holds. We satisfy the thermodynamic inequality by prescribing a kinetic model. The simplest kinetic model assumes that the solution is isotropic, and that the rates are linear in the thermodynamic forces. The combination of linearity and isotropy requires that the kinetic model should take the form:

$$
s_{i j}=2 \eta e_{i j},
$$




$$
\begin{aligned}
& \frac{\mu_{A}-F_{A}}{V_{A}}+\sigma_{m}=\beta_{A A} i_{A}+\beta_{A B} i_{B}, \\
& \frac{\mu_{B}-F_{B}}{V_{B}}+\sigma_{m}=\beta_{B A} i_{A}+\beta_{B B} i_{B} .
\end{aligned}
$$

Equations (13)-(15) constitute seven independent kinetic equations. The viscosity $\eta$ resists the change in the shape of the solution. The coefficients $\beta_{\alpha \beta}$ resist the insertion of molecules into the solution-that is, $\beta_{\alpha \beta}$ resist dilation and swap.

We assume the Onsager reciprocity, $\beta_{B A}=\beta_{A B}$. The four viscosity-like coefficients $\eta$ and $\beta_{\alpha \beta}$ have the same molecular origin: molecules in the solution change neighbors by breaking and forming intermolecular bonds. In general, the coefficients are functions of the composition of the solution, $\eta(\xi)$ and $\beta_{\alpha \beta}(\xi)$. The kinetic model (13)-(15) reduces the thermodynamic inequality (8) to $\beta_{A A} i_{A}^{2}+2 \beta_{A B} i_{A} i_{B}+\beta_{B B} i_{B}^{2}+\eta e_{i j} e_{i j} \geq 0$. The quadratic form is positive-definite for any combination of the seven independent rates $\left(i_{A}, i_{B}, e_{i j}\right)$ if and only if $\eta>0, \beta_{A A}>0, \beta_{B B}>0$ and $\beta_{A A} \beta_{B B}-\beta_{A B}^{2}>0$.

Our kinetic model generalizes Newton's model of viscosity. Note that our model differs from the model of compressible viscosity. We have assumed that molecules in the solution are incompressible, so that a change in volume of the solution is entirely due to the exchange of molecules between the reservoirs and the solution. In contrast, for compressible viscosity, the number of every species of molecules in an element of solution is fixed, and an increase in the volume of the element is entirely due to the reduction in density. Our model here generalizes a model for pure substances (Li et al., 2014) and a theory of reactive flow (Brassart and Suo, 2012; Brassart and Suo, 2013).

As noted in the Introduction, many existing theories of concurrent diffusion and deformation assume local chemical equilibrium, but allow nonequilibrium process of creep. The 
assumption of local chemical equilibrium corresponds to $F_{A}(\xi)-\sigma_{m} V_{A}(\xi)=\mu_{A}$ and $F_{B}(\xi)-\sigma_{m} V_{B}(\xi)=\mu_{B}$. The injection of two species of molecules is equivalent to a combination of dilation and swap. The nonequilibrium process of creep corresponds to shear, $s_{i j}=2 \eta e_{i j}$.

Under the assumption of local chemical equilibrium, any change in the chemical potential in the reservoirs would instantaneously change the composition and hydrostatic stress in the solution. Whereas the assumption is adequate when the local chemical processes are fast compared to diffusion, this assumption is unrealistic when creep limits mixing. During viscous mixing, the solution shears, dilates, and swaps concurrently. All three modes break and form intermolecular bonds. There is no reason to hold the bias that dilation and swap are much faster than shear. Our kinetic model places the three modes on equal footing, as distinct, concurrent, nonequilibrium processes.

The kinetic model of viscous mixing contains a time scale. The left side of (14) and (15) contains thermodynamic forces to restore equilibrium, and the right side contains rates of injection. The kinetic model of viscous mixing is thus analogous to that of viscoelasticity. The coefficients $\eta$ and $\beta_{\alpha \beta}$ have the dimension of viscosity, (stress)(time). The partial free energy $F_{A}$ has the dimension of energy per molecule, which is on the order of $k T$. We obtain a time scale for viscous mixing, $\tau_{C}=\eta \Omega / k T$. Here we have used the viscosity to define the time scale; we can of course use any viscosity-like coefficient $\beta_{\alpha \beta}$, as well as their combinations. As noted in Introduction, the time needed to homogenize a solution by viscous mixing is independent of the length scale of inhomogeneity.

For later applications, we also write the kinetic model in a different form. Equations (4), (14) and (15) constitute a linear map between $\left(i_{A}, i_{B}, \sigma_{m}\right)$ and $\left(\zeta_{A}, \zeta_{B}, d_{k k}\right)$. Solving for $i_{A}, i_{B}$ and $\sigma_{m}$, we obtain that 


$$
\begin{gathered}
i_{A}=\frac{\beta_{B B}-\beta_{A B}}{\alpha} d_{k k}+\frac{\mu_{A}-F_{A}}{\alpha V_{A}}-\frac{\mu_{B}-F_{B}}{\alpha V_{B}}, \\
i_{B}=\frac{\beta_{A A}-\beta_{A B}}{\alpha} d_{k k}-\frac{\mu_{A}-F_{A}}{\alpha V_{A}}+\frac{\mu_{B}-F_{B}}{\alpha V_{B}}, \\
\sigma_{m}=\tilde{\beta} d_{k k}-\gamma .
\end{gathered}
$$

Here we introduce several functions of composition:

$$
\begin{gathered}
\alpha=\beta_{A A}+\beta_{B B}-2 \beta_{A B}, \\
\tilde{\beta}=\frac{\beta_{A A} \beta_{B B}-\beta_{A B}^{2}}{\alpha}, \\
\gamma=\frac{\left(\beta_{B B}-\beta_{A B}\right)}{\alpha} \frac{\mu_{A}-F_{A}}{V_{A}}+\frac{\left(\beta_{A A}-\beta_{A B}\right)}{\alpha} \frac{\mu_{B}-F_{B}}{V_{B}} .
\end{gathered}
$$

These equations express $i_{A}, i_{B}$ and $\sigma_{m}$ in terms of the composition $\xi$ and the rate of dilation $d_{k k}$

2.6 Steady states

Consider a solution in chemical contact with reservoirs of fixed chemical potentials $\mu_{A}$ and $\mu_{B}$, and subject to a fixed state of stress $\sigma_{i j}$. Starting with an initial composition, $\xi(\mathrm{o})$, the solution evolves its composition in time, $\xi(t)$ according to equation (3), (14) and (15). The solution in general cannot reach a state of equilibrium. That is, given $\mu_{A}, \mu_{B}$, and $\sigma_{m}$, in general we cannot find a composition $\xi$ to satisfy both conditions of equilibrium (11)-(12), $\mu_{A}=F_{A}(\xi)-\sigma_{m} V_{A}(\xi)$ and $\mu_{B}=F_{B}(\xi)-\sigma_{m} V_{B}(\xi)$. Equations (11)-(12) constitute a system of two equations for one unknown, the equilibrium composition. For arbitrary values of chemical potentials and means stress, this system does not have a solution. A trivial exception is that the 
two reservoirs coincide with the solution of a fixed composition, and that the state of stress vanishes.

Although true equilibrium cannot be reach in general, the solution always approaches a steady state of constant composition $\xi$. The steady-state is not necessarily a true equilibrium state, as injection of species and deformations may continue indefinitely. The steady-state composition can be calculated from the kinetic equations in the following way. In the steady state, $\dot{\xi}=0$, so that the solution maintains a constant composition, $\xi$, while the reservoirs inject molecules into the solution at a fixed ratio, $\dot{n}_{A} / \dot{n}_{B}=(1-\xi) / \xi$, according to equation (3). The two kinetic equations (14) and (15) become that

$$
\begin{aligned}
& \frac{\mu_{A}-F_{A}(\xi)}{V_{A}(\xi)}+\sigma_{m}=\kappa_{A}(\xi) d_{k k}, \\
& \frac{\mu_{B}-F_{B}(\xi)}{V_{B}(\xi)}+\sigma_{m}=\kappa_{B}(\xi) d_{k k},
\end{aligned}
$$

where $\kappa_{A}=(1-\phi) \beta_{A A}+\phi \beta_{A B}$ and $\kappa_{B}=(1-\phi) \beta_{A B}+\phi \beta_{B B}$ are two types of dilation viscosities, and $\phi=\xi V_{B}(\xi) /\left[(1-\xi) V_{A}(\xi)+\xi V_{B}(\xi)\right]$ is the volume fraction of species B in the solution.

When $\mu_{A}, \mu_{B}$, and $\sigma_{m}$ are specified, (22) and (23) do predict a steady state. Eliminate $d_{k k}$ from (22) and (23), and we obtain that

$$
\kappa_{A} \frac{F_{B}}{V_{B}}-\kappa_{B} \frac{F_{A}}{V_{A}}=\kappa_{A} \frac{\mu_{B}}{V_{B}}-\kappa_{B} \frac{\mu_{A}}{V_{A}}+\left(\kappa_{A}-\kappa_{B}\right) \sigma_{m} .
$$

This is an algebraic equation for the steady-state composition. We assume that $V_{A}>0, V_{B}>0$, and all the kinetic coefficients are finite. From the thermodynamic theory of solution, $F_{A}(\mathrm{o})=F_{B}(1)=0$ and $F_{A}(1)=F_{B}(\mathrm{o})=-\infty$ (Section 5 ). The thermodynamic inequality requires 
that $\beta_{A A}>0$ and $\beta_{B B}>0$. The left side of (24) approaches $-\infty$ when $\xi \rightarrow 0$, and approaches $+\infty$ when $\xi \rightarrow 1$. By the continuity of the free energy function, the left side of (24) spans all real numbers, so that at least one solution of (24) exists for any given values of $\mu_{A}, \mu_{B}$, and $\sigma_{m}$. The injection rates, rate of dilation and rate of shear can be calculated from equations (14)-(15), (3) and (13), and do not necessarily vanish during steady-state.

In a steady state, the kinetic model gives seven independent algebraic equations, (13), (22), and (23), among 15 variables, $\xi, d_{i j}, \sigma_{i j} \mu_{A}, \mu_{B}$. The algebraic equations are nonlinear in the composition $\xi$, but are linear in the other 14 variables. After specifying eight variables, we can use the kinetic model to calculate the other seven variables. In the above, we have fixed the chemical potentials of the two species of molecules in the reservoirs and the six components of stress, and then use the kinetic model to calculate the composition and the six components of the rate of deformation. Of course, we can also achieve a steady state by specifying other combinations of eight variables.

\section{Theory of inhomogeneous viscous mixing}

We now consider a body of inhomogeneous solution (Fig. 4). Each small piece of the body evolves through a sequence of homogeneous states, as described above. Different pieces in the body communicate through the compatibility of deformation, balance of forces, and conservation of molecules. 


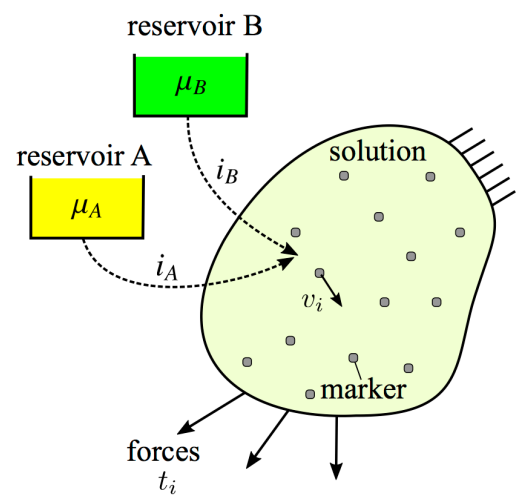

Fig. 4. A body of binary solution is in chemical contact with reservoirs of species A and B, and is subject to external forces acting on its boundary. Dispersed in the solution are markers moving at the velocity $v_{i}$.

\subsection{Compatibility of deformation}

During mixing, each piece in the body does not preserve the number and identity of molecules. The notion of a "material particle" in continuum mechanics becomes questionable. Following Darken (1948) and Stephenson (1988), we imagine to disperse in the solution a field of markers, such as those commonly used in experimental fluid mechanics to visualize flows. Individual markers are small enough so that they represent the flow of the solution with fidelity, but are large enough so that they do not participate in mixing themselves.

We use markers to identify pieces of the body. Let $\mathbf{x}$ be the coordinate of a place in space, and $v_{i}(\mathbf{x}, t)$ be the field of velocity of the makers. The rate of deformation relates to the field of velocity as

$$
d_{i j}=\frac{1}{2}\left(\frac{\partial v_{i}}{\partial x_{j}}+\frac{\partial v_{j}}{\partial x_{i}}\right)
$$

When molecules are inserted into the solution between two markers, the two markers drift apart. Thus, the relative movements of the markers describe the deformation of the solution. 
We next consider the velocity of markers near a boundary. Examples include a free boundary of the solution, an interface between the solution and a rigid solid, and an interface between two regions of the solution of different composition. We have assumed that diffusion is so fast that chemical potential of each species of molecules is the same everywhere in the system. To be consistent, we now further assume that during mixing molecules do not append to each boundary, but rapidly diffuse throughout the solution. So long as the rate of deformation is finite, the velocity of a marker in the solution, near the boundary, is the same as the velocity of the boundary. Thus, the field of marker velocity is continuous.

Our Eulerian formulation based on marker velocity differs from the classical hydrodynamic theory for multiple species, which identifies the bulk velocity of the solution as the barycentric velocity (the mass-weighted average of the components velocity) (de Groot and Mazur, 1984; Landau and Lifshitz, 1987). It then follows that the rates of injections of each species cannot be prescribed independently in order to preserve a constant mass within a material element. In the case of a single species, the classical hydrodynamic theory cannot differentiate between creep and self-diffusion, as noted by Li et al. (2014). In contrast, small pieces defined by marker do not need to maintain a constant mass. Rather, markers drift apart as molecules are injected into the piece (dilation), or as the piece changes shape under shear. The use of marker velocity is thus consistent with the theory of homogeneous states described in Section 2. The notion of marker velocity was first introduced by Darken (1948) to describe the Kirkendall effect. A complete theory of interdiffusion coupled to deformations based on the marker velocity is described by Stephenson (1988).

\subsection{Balance of forces}

Denote the field of stress in the body by $\sigma_{i j}(\mathbf{x}, t)$. Inside the body, the balance of mechanical forces requires that 


$$
\frac{\partial \sigma_{i j}}{\partial x_{j}}+b_{i}=0
$$

where $b_{i}$ is the density of body force. The balance of forces further relates the traction acting on a plane in the body to the state of stress:

$$
t_{i}=\sigma_{i j} n_{j}
$$

where $t_{i}$ is the traction acting on the plane, and $n_{j}$ is the unit vector normal to the plane.

\subsection{Conservation of molecules}

In an inhomogeneous field, the composition is a function of place and time, $\xi(\mathbf{x}, t)$.

Consequently, we replace $\dot{\xi}$ in (3) by the rate of change in the composition around a marker, $\partial \xi(\mathbf{x}, t) / \partial t+v_{i} \partial \xi(\mathbf{x}, t) / \partial x_{i}$, and obtain that

$$
\frac{\partial \xi}{\partial t}+v_{i} \frac{\partial \xi}{\partial x_{i}}=\frac{(1-\xi) \Omega}{V_{B}} i_{B}-\frac{\xi \Omega}{V_{A}} i_{A} .
$$

Thus, the rate of change in composition at a place is due to the movements of the makers, as well as the injection of the molecules.

As mentioned in the Introduction, we assume that diffusion is fast, so that the chemical potentials of the two species in the solution are uniform in the solution, but in general can change in time, $\mu_{A}(t)$ and $\mu_{B}(t)$. So far we have assumed that the values of chemical potentials are prescribed by the values in the reservoirs. This is true when the body is in chemical contact with the reservoirs. However, when the body is disconnected from the reservoirs, molecules can still diffuse rapidly from one piece of the body to another, so that the chemical potentials $\mu_{A}(t)$ and $\mu_{B}(t)$ are still uniform, but their values must be determined by solving the boundary-value problem. The total numbers of the two species are given by 


$$
n_{A}=\int \frac{(1-\xi) d V}{\Omega(\xi)}, \quad n_{B}=\int \frac{\xi d V}{\Omega(\xi)}
$$

The integrals extend over the volume of the body. For a body disconnected from the reservoirs, the total number of each species in the body is independent of time. This constraint provides the equations needed to determine the chemical potentials $\mu_{A}(t)$ and $\mu_{B}(t)$.

3.4 Governing equations to evolve the field of marker velocity and the field of composition

We combine various ingredients described before into equations that evolve four fields, $v_{i}(\mathbf{x}, t)$ and $\xi(\mathbf{x}, t)$. Recall that $\sigma_{i j}=s_{i j}+\sigma_{m} \delta_{i j}$, and that $s_{i j}$ and $\sigma_{m}$ relate to the marker velocity and composition through (13) and (18). Inserting these relations into (26), we obtain that

$$
\frac{\partial}{\partial x_{j}}\left(\eta\left(\frac{\partial v_{i}}{\partial x_{j}}+\frac{\partial v_{j}}{\partial x_{i}}\right)\right)+\frac{\partial}{\partial x_{i}}\left(\left(\tilde{\beta}-\frac{2}{3} \eta\right) \frac{\partial v_{k}}{\partial x_{k}}\right)-\frac{d \gamma}{d \xi} \frac{\partial \xi}{\partial x_{i}}+b_{i}=0
$$

This equation generalizes the equation for the Stokes flow. A gradient in composition acts as a body force through the term $-(d \gamma / d \xi) \partial \xi / \partial x_{i}$. Given a field of composition $\xi(\mathbf{x}, t)$, equation (30) governs the field of velocity $v_{i}(\mathbf{x}, t)$. Equation (30) does not contain time explicitly, and serves as three governing equations for a boundary-value problem. The boundary conditions can be either prescribed velocity or prescribed traction.

Inserting (16) and (17) into (28), we obtain that

$$
\begin{aligned}
\frac{\partial \xi}{\partial t}= & -v_{i} \frac{\partial \xi}{\partial x_{i}} \\
& +\frac{(1-\xi) \Omega}{V_{B}}\left(\frac{\beta_{A A}-\beta_{A B}}{\alpha} d_{k k}-\frac{\mu_{A}-F_{A}}{\alpha V_{A}}+\frac{\mu_{B}-F_{B}}{\alpha V_{B}}\right) \\
& -\frac{\xi \Omega}{V_{A}}\left(\frac{\beta_{B B}-\beta_{A B}}{\alpha} d_{k k}+\frac{\mu_{A}-F_{A}}{\alpha V_{A}}-\frac{\mu_{B}-F_{B}}{\alpha V_{B}}\right)
\end{aligned}
$$


Given fields of composition $\xi(\mathbf{x}, t)$ and marker velocity $v_{i}(\mathbf{x}, t)$, equation (31) updates the field of composition in time. As noted before, the chemical potentials are uniform in the body. When the body is connected to the reservoirs, the chemical potentials are prescribed. When the body is disconnected from the reservoirs, the chemical potentials are determined by the conservation of the numbers of the two species.

\section{On the experimental determination of the viscosity-like coefficients}

Consider homogeneous and time-dependent viscous mixing. Up to a difference in rigidbody motion, the homogenous rate of deformation $d_{i j}(t)$ corresponds to an affine field of marker velocity, $v_{i}(\mathbf{x}, t)=d_{i j}(t) x_{j}$. Any state of homogeneous stress $\sigma_{i j}(t)$ balances forces in the solution, assuming a zero body force. The theory of viscous mixing relates eight rates $\left(i_{A}, i_{B}, d_{i j}\right)$

to eight driving forces $\left(\mu_{A}, \mu_{B}, \sigma_{i j}\right)$ through eight equations: the condition of molecular incompressibility (4), and the seven kinetic relations (13)-(15). We next consider several types of behavior that lend themselves to the experimental determination of the viscosity-like coefficients.

\subsection{Creep experiment}

When the solution is isolated from the reservoirs but is subject to stresses, the solution undergoes purely deviatoric creep, which preserves the number of each species of molecules in the solution, and keeps the composition $\xi$ fixed. Purely deviatoric creep can be used to determine the shear viscosity as a function of composition, $\eta(\xi)$, as commonly done in experimental rheology. 
4.2 Steady-state, free swelling experiment

A steady state may be a convenient experimental setup to measure the viscosity-like coefficients. For example, in contact with reservoirs of fixed chemical potentials $\mu_{A}$ and $\mu_{B}$, and in the absence of any stress, the solution will reach a steady state of a certain composition $\xi$ and a certain rate of dilation $d_{k k}$. Equations (22) and (23) enable us to determine $\kappa_{A}$ and $\kappa_{B}$ for the specific composition. We then repeat the experiment with reservoirs of different values of the chemical potentials, so that the solution reaches a steady state of a different composition. Such experiment will allow us to measure the two viscosities of dilation as functions of composition, $\kappa_{A}(\xi)$ and $\kappa_{B}(\xi)$. They correspond to the two combined coefficients, $\kappa_{A}=(1-\phi) \beta_{A A}+\phi \beta_{A B}$ and $\kappa_{B}=(1-\phi) \beta_{A B}+\phi \beta_{B B}$. However, to determine all three viscosity-like coefficients, $\beta_{A A}(\xi), \beta_{A A}(\xi)$, and $\beta_{A B}(\xi)$, we will need a third independent measurement, which must be done in a transient experiment.

\subsection{Relaxation toward a state of equilibrium}

As an example of transient experiment, consider pure swap. A container with rigid and permeable walls is filled with a solution, and is in contact with reservoirs of fixed chemical potentials $\mu_{A}$ and $\mu_{B}$. We assume that the container is "sufficiently small" so that the rate of deformation and composition of the solution can be considered macroscopically uniform within the container. The rigid walls suppress deformation, $d_{i j}=0$. As the solution and the reservoirs exchange molecules, the solution evolves the composition $\xi(t)$ and the hydrostatic stress $\sigma_{m}(t)$.

In pure swap, the two rates of volumetric injection counteract, $i_{A}=-i_{B}$. Inserting (16) and (17) into (3), we obtain that 


$$
\dot{\xi}=\frac{[\Omega(\xi)]^{2}}{\alpha(\xi) V_{A}(\xi) V_{B}(\xi)}\left[\frac{\mu_{B}-F_{B}(\xi)}{V_{B}(\xi)}-\frac{\mu_{A}-F_{A}(\xi)}{V_{A}(\xi)}\right] .
$$

This ordinary differential equation evolves the composition in time, $\xi(t)$.

After some time, the solution reaches a state of thermodynamic equilibrium, with the composition and hydrostatic stress determined by (11) and (12). Let $\xi_{\text {eq }}$ be the composition of the solution in equilibrium with the reservoirs of the fixed chemical potentials $\mu_{A}$ and $\mu_{B}$. Linearizing (32) around the state of equilibrium, we obtain an ordinary equation of form $\dot{\xi}=\left(\xi_{e q}-\xi\right) / \tau$. The relaxation time $\tau$ depends on $\xi_{e q}$, and is proportional to the viscosity-like coefficient $\alpha$. The linear ordinary differential equation gives that $\xi_{\text {eq }}-\xi(t)=\left(\xi_{\text {eq }}-\xi(\mathrm{o})\right) \exp (-t / \tau)$. Experimentally, we can start with a binary solution near a state of thermodynamic equilibrium, $\xi_{e q}$, and measure the relaxation time, which gives a value of the viscosity-like coefficient $\alpha$ at $\xi_{\text {eq }}$. By adjusting the chemical potentials of the reservoir and repeat the experiment around variations states of equilibrium, we can determine the function $\alpha(\xi)$. The combination of the coefficients $\alpha(\xi), \kappa_{A}(\xi)$ and $\kappa_{B}(\xi)$ allows us to identify the three viscosity-like coefficients at a fixed composition.

\section{Ideal mixing of molecules of unequal sizes and nonzero volume of mixing}

To give numerical examples in later sections, here we formulate a model of ideal mixing of molecules of unequal sizes and nonzero volume of mixing. This model recovers the model of ideal mixing of molecules of similar sizes. The model also recovers the model of polymer blends, where the volume of mixing is absent (Huggins, 1941; Flory, 1942).

By ideal mixing we mean that, when two pure substances A and B mix and form a binary solution, every molecule retains its number of conformations. The mixing, however, increases 
the volume in which every molecule can explore (Fig. 5). In each pure substance and in the solution, every molecule is free to explore the entire volume. The solution provides a larger volume for every molecule to explore than the two pure substances. This difference in volume results in the entropy of ideal mixing, as calculated below.
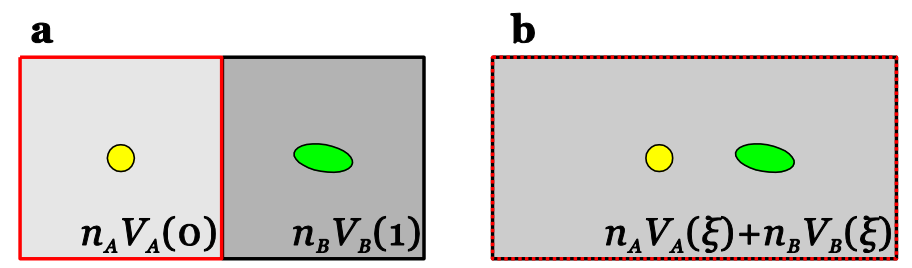

Fig. 5. During ideal mixing, each individual molecule retains the same number of configurations, but can access an increased volume. (a) Before mixing, each molecule of species A can only access a volume $n_{A} V_{A}(\mathrm{o})$, and each molecule of species B can only access a volume $n_{B} V_{B}(1)$. (b) After mixing, molecules of A and B can access a larger volume $n_{A} V_{A}(\xi)+n_{B} V_{B}(\xi)$.

Recall that the composition of the solution is $\xi=n_{B} /\left(n_{A}+n_{B}\right)$, and that $V_{A}(\xi)$ and $V_{B}(\xi)$ are partial volumes of the molecules in the solution. When $n_{A}$ molecules of species A are in the state of pure substance, the volume of the pure substance is $n_{A} V_{A}(\mathrm{o})$. Because every molecule is free to explore the entire volume, the number of configurations of the pure substance scales as $\left[n_{A} V_{A}(\mathrm{o})\right]^{n_{A}}$. Similarly, when $n_{B}$ molecules of species B are in the state of pure substance, the volume of the pure substance is $n_{B} V_{B}(1)$, and the number of configurations of the pure substance scales as $\left[n_{B} V_{B}(1)\right]^{n_{B}}$. When the two substances mix, the volume of the solution is $V=n_{A} V_{A}(\xi)+n_{B} V_{B}(\xi)$, and the number of configurations of the solution scales as $\left[n_{A} V_{A}(\xi)+n_{B} V_{B}(\xi)\right]^{n_{A}+n_{B}}$. We adopt the convention of dimensionless entropy, defined as the 
logarithm of the number of configurations. Consequently, when the two substances mix and form the binary solution, the entropy changes by

$$
S_{\text {mix }}=\log \frac{\left[n_{A} V_{A}(\xi)+n_{B} V_{B}(\xi)\right]^{n_{A}+n_{B}}}{\left[n_{A} V_{A}(\mathrm{o})\right]^{n_{A}}\left[n_{B} V_{B}(1)\right]^{n_{B}}} .
$$

This model allows molecules of arbitrary size and shape, and allows arbitrary volume of mixing.

By ideal mixing we also stipulates that the energy of mixing vanishes, so that the Helmholtz free energy is $F=-k T S_{\text {mix }}$. We adopt the convention that sets the Helmholtz free energy of each pure substance to be zero. Thus, the partial Helmholtz free energies $F_{A}=\partial F\left(n_{A}, n_{B}\right) / \partial n_{A}$ and $F_{B}=\partial F\left(n_{A}, n_{B}\right) / \partial n_{B}$ are

$$
\begin{aligned}
& F_{A}(\xi)=k T\left[\log \frac{(1-\xi) V_{A}(\mathrm{o})}{(1-\xi) V_{A}(\xi)+\xi V_{B}(\xi)}+\frac{\xi\left(V_{B}(\xi)-V_{A}(\xi)\right)}{(1-\xi) V_{A}(\xi)+\xi V_{B}(\xi)}\right], \\
& F_{B}(\xi)=k T\left[\log \frac{\xi V_{B}(1)}{(1-\xi) V_{A}(\xi)+\xi V_{B}(\xi)}+\frac{(1-\xi)\left(V_{A}(\xi)-V_{B}(\xi)\right)}{(1-\xi) V_{A}(\xi)+\xi V_{B}(\xi)}\right] .
\end{aligned}
$$

\section{Examples of homogeneous viscous mixing}

Assume the following properties: $V_{A}=10 V_{B}, \beta_{A A}=2 \eta, \beta_{B B}=\eta$, and $\beta_{A B}=0$; they are all independent of the composition. The solution is in chemical contact with a reservoir of the two species of molecules of fixed chemical potentials $\mu_{A} / k T=-1$ and $\mu_{B} / k T=-0.1$. The initial composition of the solution is $\xi(\mathrm{o})=0.01$.

\subsection{Swap}

Consider first a case of pure swap. The solution fills a box of rigid and permeable walls, and the box is immersed in a reservoir of the two species of molecules of fixed chemical 
potentials. Integrating the ordinary differential equation (32), we obtain the composition as a function of time (Fig. 6a). We have assumed a low value of initial concentration of $\mathrm{B}$ in the solution, and a relatively high value of the chemical potential of B in the reservoir. Consequently, the solution swaps two species of molecules with the reservoir, exudes species A, and imbibes species B. Once the function $\xi(t)$ is obtained, (18) determines the hydrostatic stress as a function of time (Fig. 6b). We have assumed that diffusion is fast, and instantaneously broadcasts the chemical potentials of the molecules in the reservoir into the solution. Consequently, the hydrostatic stress in the solution is nonzero at time zero. As the solution and the reservoir swap the two species of molecules, the hydrostatic stress in the solution reduces in magnitude. After some time of swapping, the solution attains a state of thermodynamic equilibrium. In the state of equilibrium, the composition and hydrostatic stress are determined by algebraic equations (11) and (12).

a)

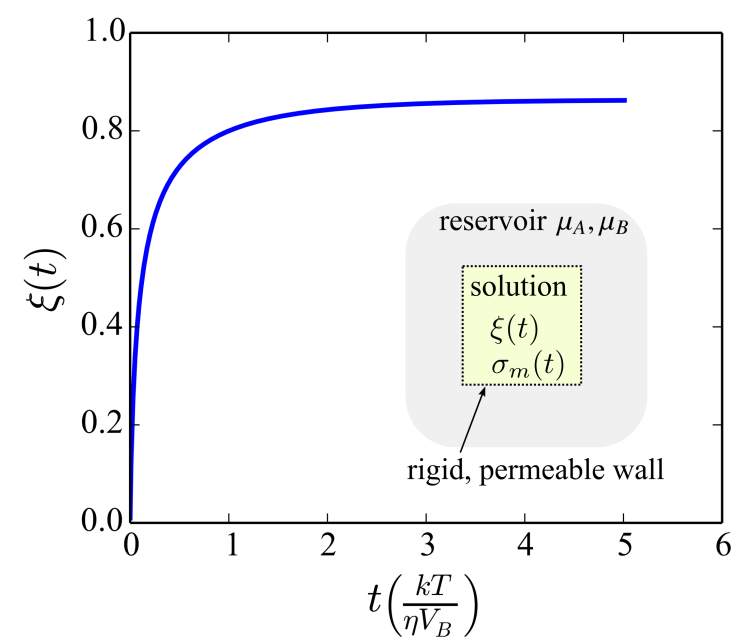

b)

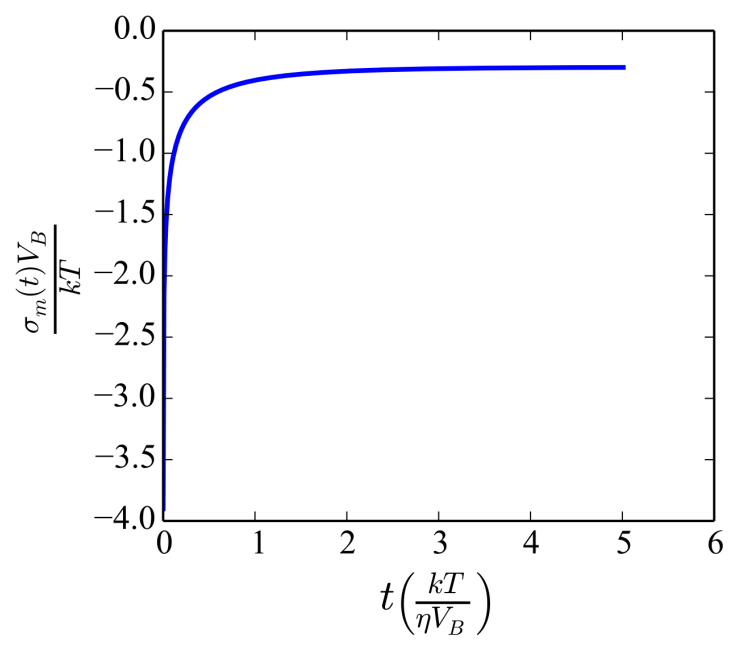

Fig. 6. A box of rigid and permeable walls is filled with a solution, and is immersed in a reservoir of fixed chemical potentials. (a) The composition as a function of time. (b) The hydrostatic stress as a function of time. 
6.2 Swelling of a solution attached to a substrate

Next consider a solution in chemical contact with a reservoir of fixed chemical potentials $\mu_{A}$ and $\mu_{B}$, and bonded to a rigid substrate (Fig. 7a). The surface of the substrate lies in the $(x, y)$ plane. Imbibing molecules from the reservoir, the solution grows in thickness, $d_{z z} \neq 0$, and develops a state of equal-biaxial stress, $\sigma_{x x}=\sigma_{y y} \neq 0$. All other components of the rate of deformation and stress vanish. The mean stress is $\sigma_{m}=2 \sigma_{x x} / 3$, and a component of the deviatoric stress is $s_{x x}=\sigma_{x x} / 3$. The rate of dilation is $d_{k k}=d_{z z}$, and a component of the deviatoric rate of deformation is $e_{x x}=-d_{z z} / 3$. Recall the kinetic model $s_{x x}=2 \eta e_{x x}$, and we obtain that $\sigma_{x x}=-2 \eta d_{z z}$ and $\sigma_{m}=-4 \eta d_{z z} / 3$.

a)

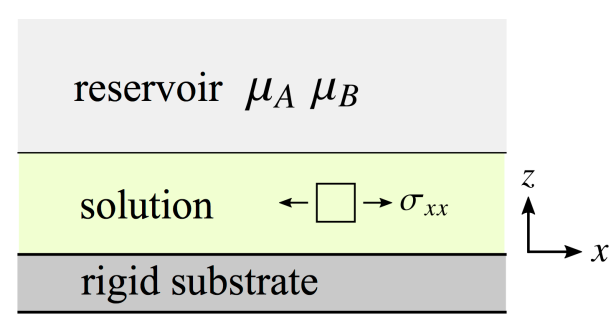

c) b)

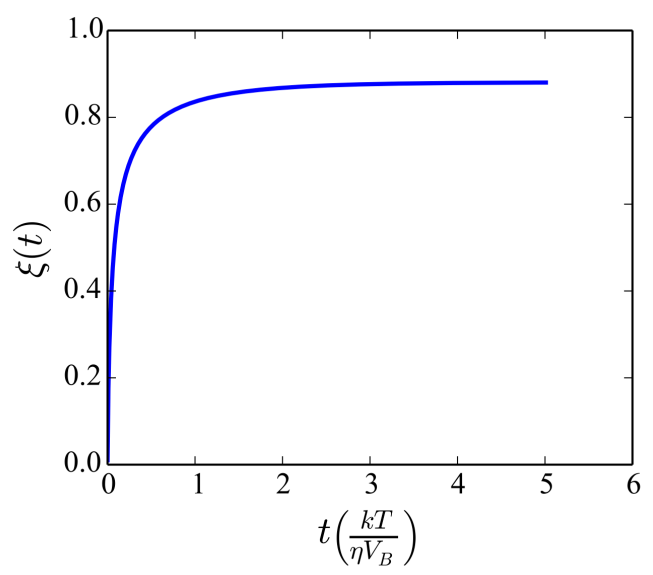

d) 

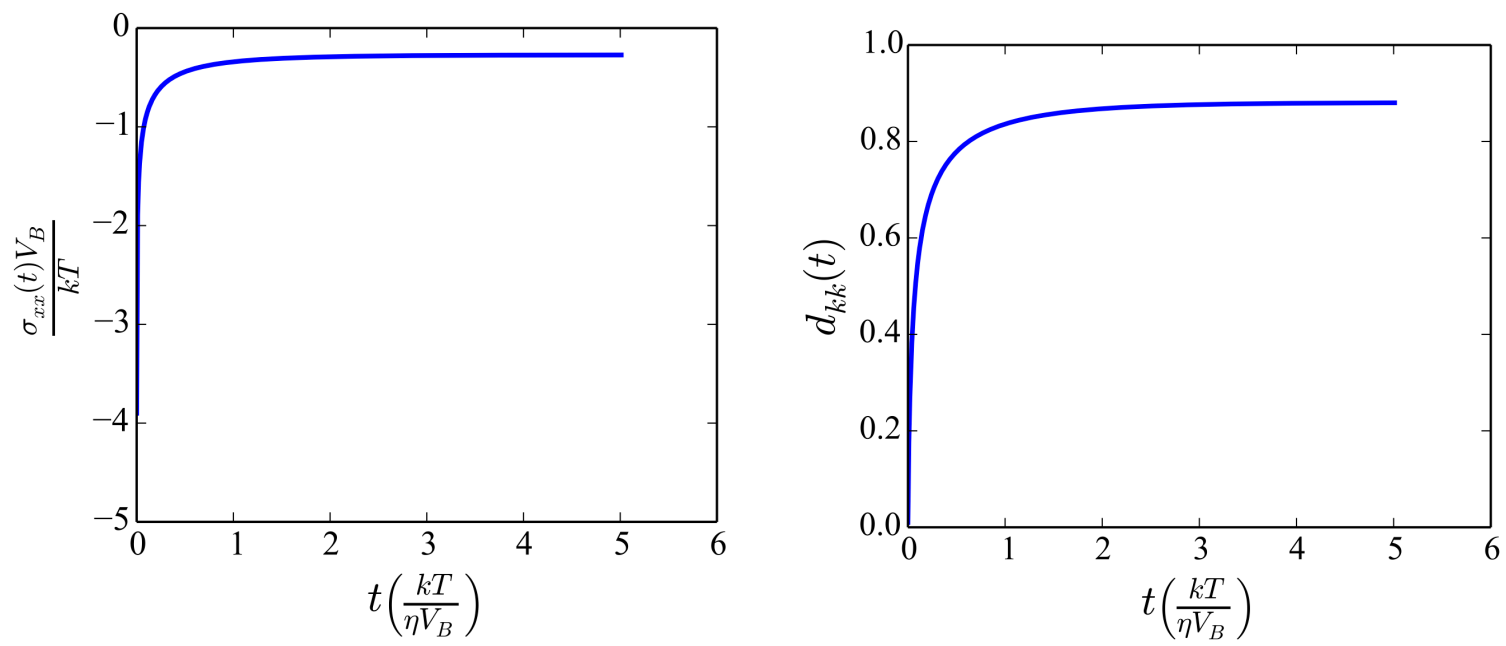

Fig 7. A solution is in chemical contact with reservoirs of two species of molecules, and is attached to a rigid substrate. The solution increases in thickness and develops a state of equal biaxial stress. (a) Schematic of the setup. (b) The composition as a function of time. (c) The equal biaxial stress as a function of time. (d) Rate of dilation as a function of time.

Inserting these relations in the incompressibility condition (4) gives the mean stress as a function of the injection rates:

$$
\boldsymbol{\sigma}_{m}=-\frac{4 \boldsymbol{\eta}}{3}\left(i_{A}+i_{B}\right)
$$

Inserting this expression into the kinetic model (14)-(15) yields:

$$
\begin{gathered}
\frac{\boldsymbol{\mu}_{A}-F_{A}(\boldsymbol{\xi})}{V_{A}}=\left(\boldsymbol{\beta}_{A A}+\frac{4 \boldsymbol{\eta}}{3}\right) i_{A}+\left(\boldsymbol{\beta}_{A B}+\frac{4 \boldsymbol{\eta}}{3}\right) i_{B}, \\
\frac{\boldsymbol{\mu}_{B}-F_{B}(\boldsymbol{\xi})}{V_{A}}=\left(\boldsymbol{\beta}_{A B}+\frac{4 \boldsymbol{\eta}}{3}\right) i_{A}+\left(\boldsymbol{\beta}_{B B}+\frac{4 \boldsymbol{\eta}}{3}\right) i_{B} .
\end{gathered}
$$

At a given composition, equations (37) and (38) are two linear equations that can be solved for the two injection rates, $i_{A}$ and $i_{B}$. Using the injection rates in (3) then enables us to evolve the composition in time, as well as the stress and the rate of dilation (Fig. 7). In particular, the 
swelling of the layer is constrained by the rigid substrate, and the constraint causes a growth stress in the solution.

\section{Viscous mixing couple}

Two thin layers of the solution are attached on a rigid substrate. The two layers can exchange molecules, but are isolated from any external reservoir. Thus, the number of each species of the molecules is fixed: $n_{A}^{(1)}(t)+n_{A}^{(2)}(t)=n_{A}$ and $n_{B}^{(1)}(t)+n_{B}^{(2)}(t)=n_{B}$. The superscripts indicate the two layers, and $n_{A}$ and $n_{B}$ stand for the total numbers of molecules of the two species, which are constant independent of time. At time $t=0$, each layer is homogeneous, but the two layers have different compositions, $\xi^{(1)}(0) \neq \xi^{(2)}(0)$. As the two layers exchange molecules, the composition of each layer changes, but remains homogeneous. The two layers will approach a homogeneous, stress-free state of equilibrium, with the composition $\xi=n_{B} /\left(n_{A}+n_{B}\right)$.

Before reaching the state of equilibrium, each layer develops a state of equal-biaxial stress, so that $\sigma_{m}^{(1)}=-4 \eta\left(\xi^{(1)}\right) d_{z z}^{(1)} / 3$ and $\sigma_{m}^{(2)}=-4 \eta\left(\xi^{(2)}\right) d_{z z}^{(2)} / 3$. In each layer, (4) relates the rate of dilation to the rates of injection. The two layers have the same chemical potentials, $\mu_{A}(t)$ and $\mu_{B}(t)$. Equations (14) and (15) hold for both layers. Eliminating the chemical potentials, we obtain that

$$
\begin{aligned}
& {\left[\sigma_{m} V_{A}-F_{A}\right]^{(2)}-\left[\sigma_{m} V_{A}-F_{A}\right]^{(1)}=\left[V_{A} \frac{\beta_{A A} V_{A} \dot{n}_{A}+\beta_{A B} V_{B} \dot{n}_{B}}{V_{A} n_{A}+V_{B} n_{B}}\right]^{(2)}-\left[V_{A} \frac{\beta_{A A} V_{A} \dot{n}_{A}+\beta_{A B} V_{B} \dot{n}_{B}}{V_{A} n_{A}+V_{B} n_{B}}\right]^{(1)},} \\
& {\left[\sigma_{m} V_{B}-F_{B}\right]^{(2)}-\left[\sigma_{m} V_{B}-F_{B}\right]^{(1)}=\left[V_{B} \frac{\beta_{A B} V_{A} \dot{n}_{A}+\beta_{B B} V_{B} \dot{n}_{B}}{V_{A} n_{A}+V_{B} n_{B}}\right]^{(2)}-\left[V_{B} \frac{\beta_{A B} V_{A} \dot{n}_{A}+\beta_{B B} V_{B} \dot{n}_{B}}{V_{A} n_{A}+V_{B} n_{B}}\right]^{(1)}}
\end{aligned}
$$


Once we insert the algebraic relations indicated above, (39) and (40) become ordinary differential equations that evolve two functions $n_{A}^{(1)}(t)$ and $n_{B}^{(1)}(t)$. The chemical potentials as functions of time can also be calculated such that the total number of molecules of each species is fixed.

Similar couples are commonly used in experiments of diffusion-limited mixing. The time for diffusion-limited mixing scales with the thickness squared. However, the relaxation time for viscous mixing is independent of the thickness of the layers.

\section{An inclusion in a matrix}

Consider an inclusion in an infinite matrix. Both the inclusion and the matrix are solutions of two species of molecules A and B. At time zero, the matrix has a homogeneous composition of one value, $\xi_{\infty}$, and the inclusion has a homogeneous composition of another value, $\xi(0)$. We will show that, as time evolves, the composition will still be piecewise homogeneous. In the matrix, the composition will remain fixed at $\xi_{\infty}$ at all time. In the inclusion, the composition will evolve as a function of time, $\xi(t)$. As $t \rightarrow \infty$, the matrix and inclusion homogenize, $\xi(t) \rightarrow \xi_{\infty}$. Thus, the inclusion evolves by a combination of dilation and swap, with no shear. We will further show that the matrix is incompressible at all time, so that the matrix evolves by pure shear, without dilation and swap. The matrix is infinite, and acts like

a reservoir of a fixed chemical potentials $\mu_{A}=F_{A}\left(\xi_{\infty}\right)$ and $\mu_{B}=F_{B}\left(\xi_{\infty}\right)$. These values of chemical potentials prevail everywhere in the matrix and in the inclusion, since we assume that diffusion is fast. The size of the heterogeneity is so small that evolution of the system is limited by viscous mixing rather than by diffusion. 
We adopt a spherical coordinate system. By symmetry, the marker velocity is purely radial and is only a function of the radial coordinate and time, $v(r, t)$. Let the radius of the inclusion be $a(t)$, and the velocity of the interface be $\dot{a}(t)$. As noted in Section 3.1, the velocity of markers is continuous across the interface. On either side of the interface, it must satisfy the momentum balance equation (27), which here reduces to

$$
\frac{d^{2} v}{d r^{2}}+\frac{2}{r}\left(\frac{d v}{d r}-\frac{v}{r}\right)=0 .
$$

The marker velocity in the inclusion solution of $(41)$ is $v(r, t)=r \dot{a} / a$. The inclusion undergoes homogeneous, time-dependent dilation with rate $d_{k k}=3 \dot{a} / a$. The inclusion is in a state of homogeneous, time-dependent, hydrostatic stress, given by (18), $\sigma_{m}=\tilde{\beta}(\xi) d_{k k}-\gamma(\xi)$.

In the matrix, the field of velocity solution of (41) is

$$
v(r, t)=\dot{a}\left(\frac{a}{r}\right)^{2} .
$$

Consequently, $d_{k k}=\mathrm{o}$ and the matrix deforms by pure shear, as in the Stokes flow. According to (16)-(18), no molecules inject into the matrix, and the mean stress vanishes. According to (28), the composition in the matrix does not evolve since the composition is homogeneous and the convective term involving $\partial \xi / \partial x_{i}$ vanishes. Corresponding to this field of velocity, the radial rate of deformation is $d_{r}=\partial v / \partial r=-2 \dot{a} a^{2} / r^{3}$, and the circumferential rate of deformation is $d_{\theta}=v / r=\dot{a} a^{2} / r^{3}$. The radial and circumferential stresses in the matrix, $\sigma_{r}(r, t)$ and $\sigma_{\theta}(r, t)$, are given by the creep model (13):

$$
\sigma_{r}=-4 \eta\left(\xi_{\infty}\right) \frac{a^{2}}{r^{3}} \dot{a}, \sigma_{\theta}=2 \eta\left(\xi_{\infty}\right) \frac{a^{2}}{r^{3}} \dot{a}
$$


In the matrix, the state of stress is triaxial: one radial component $\sigma_{r}$ and two circumferential components of equal magnitude $\sigma_{\theta}$.

The inclusion dilates and swaps by absorbing molecules from the matrix, whereas the matrix evolves by pure shear and does not change composition. This non-intuitive result follows from our hypothesis of an infinite matrix and fixed chemical potentials. The matrix is able to maintain its equilibrium composition because molecules in the matrix can redistribute infinitely fast from remote areas in the matrix. In the case where the matrix has a finite size, one cannot fix the values of chemical potentials. The chemical potential must be calculated in such a way that the total number of molecules in the system is preserved, as noted in Section 3.3.

At the interface, the balance of forces requires that the radial stress in the inclusion equal that in the matrix. Equating $\boldsymbol{\sigma}_{m}^{(i)}=\tilde{\boldsymbol{\beta}}(\boldsymbol{\xi}) d_{k k}^{(i)}-\boldsymbol{\gamma}(\boldsymbol{\xi})$ and $\boldsymbol{\sigma}_{r}^{(m)}=-4 \boldsymbol{\eta}\left(\boldsymbol{\xi}_{\infty}\right) d_{k k}^{(i)} / 3$ (the superscripts refer to the inclusion and the matrix), we obtain the dilation in the inclusion:

$$
d_{k k}^{(i)}=\frac{\gamma(\xi)}{\tilde{\beta}(\xi)+4 \eta\left(\xi_{\infty}\right) / 3} .
$$

Inserting (44) into (28), we obtain an ordinary differential equation that evolves the composition of the inclusion, $\xi(t)$. Because the composition in the inclusion is homogeneous, in (28) the convective term involving $\partial \xi / \partial x_{i}$ vanishes. Once $\xi(t)$ is determined, the equations listed above determine the fields of stress and velocity everywhere, as well as the dilation in the inclusion. The radius of inclusion is determined from $d_{k k}^{(i)}=3 \dot{a} / a$. Thus,

$$
\log \frac{a(t)}{a(\mathrm{o})}=\frac{1}{3} \int_{\mathrm{o}}^{t} d_{k k}^{(i)}(\xi) d t
$$

We illustrate the solution of the inclusion problem by considering the same material properties as those in Section 6. We assume that the composition in the matrix is $\xi_{\infty}=0.5$ and the initial composition of the inclusion is $\xi(0)=0.01$. As expected, the composition of the 
inclusion increases in time, and approaches that of the matrix (Fig. 8a). The inclusion dilates somewhat in time (Fig. 8b). In this case, our numerical simulation indicates that the inclusion swaps more substantially than dilates. The mean stress in the inclusion starts at a finite negative value and vanishes in long time (Fig. 8c). At any given time, the marker velocity is continuous across the interface, but its first derivative is not (Fig. 8d). 
a)

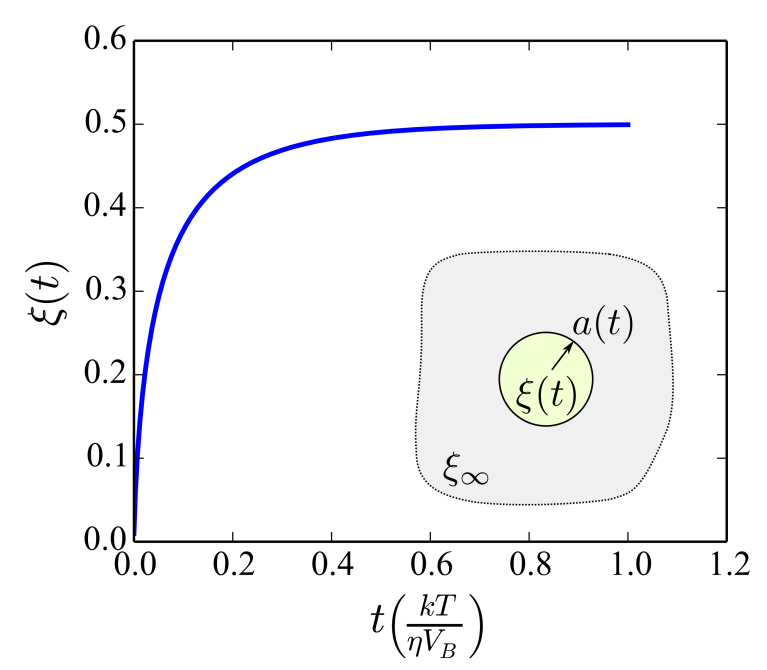

c)

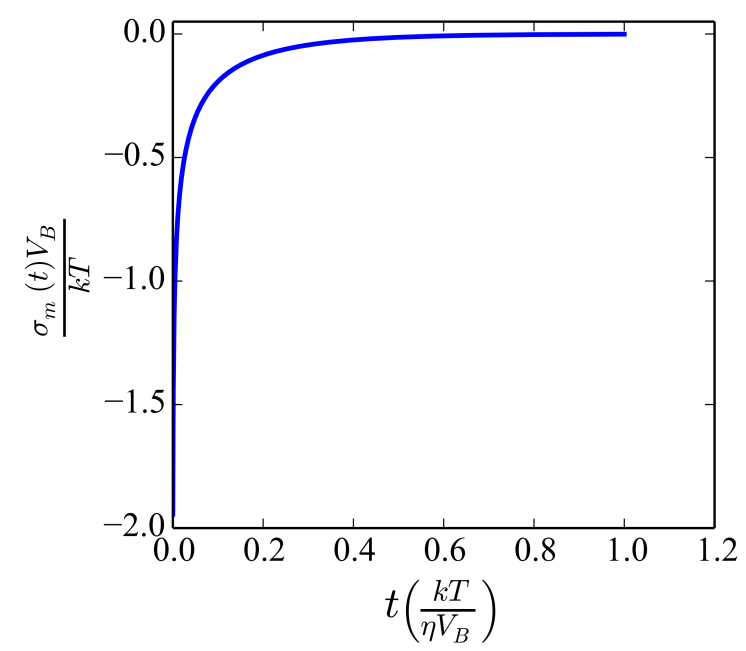

b)

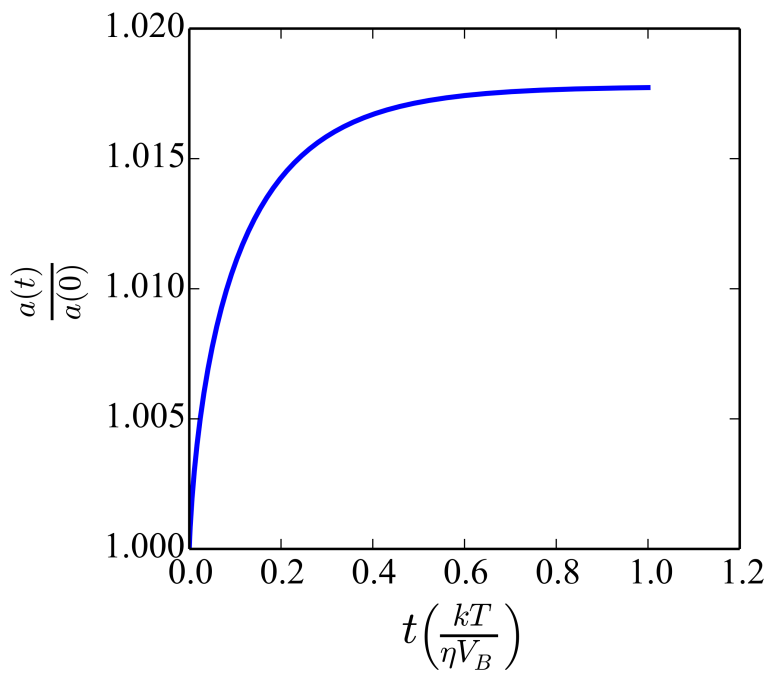

d)

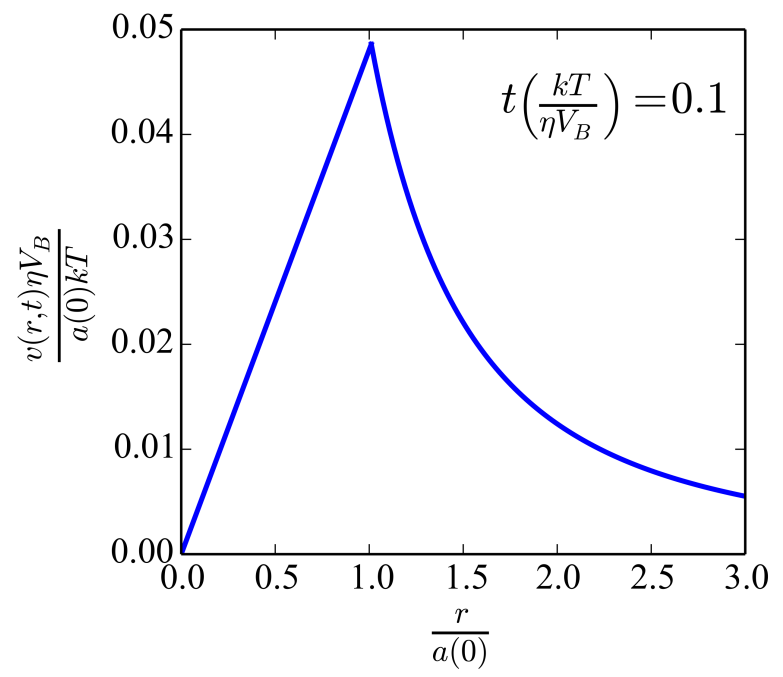

Fig. 8. Homogenization of a spherical inclusion in an infinite matrix. The evolution of (a) the composition, (b) the radius, and (c) the mean stress of the inclusion as functions of time. (d) The profile of the velocity in the inclusion and matrix at a given time. 


\section{Concluding remarks}

This paper formulates a theory of mixing when the length scale of inhomogeneity is small compared to the poroviscous length. In this limit, molecules diffuse so fast that the chemical potential of each species of molecules is homogeneous, and the rate of mixing is limited not by long-range migration of molecules, but by local rearrangement of molecules. When viscous mixing prevails, the time for homogenization is independent of the length scale of inhomogeneity. We specify the kinematics of local rearrangement by two rates of injection and five rates of deviatoric deformation. We associate the seven rates with seven thermodynamic forces, and propose a kinetic model of four independent viscosity-like coefficients. Our model places various modes of rearrangements-shear, dilation, and swap-on equal footing, as distinct, concurrent, nonequilibrium processes. The four viscosity-like coefficients can be determined by various steady and transient experiments. We also derive a thermodynamic model of ideal mixing of molecules of unequal sizes and nonzero volume of mixing. The theory is illustrated using several examples, including the development of growth stress, the homogenization of a bilayer, and the disappearance of an inclusion in a matrix.

Our theory of viscous mixing is relevant to interpret recent experiments on physical gels. As an example, the two processes of poroviscous and viscous relaxation have been highlighted in recent stress relaxation experiments on alginate (Zhao et al. 2010; Gentile et al. 2013). In these experiments, the diameter of the sample is of the $\mathrm{cm}$ size, and the diffusion coefficient of water through in alginate was estimated as $D_{\text {water }}=10^{-8} \mathrm{~m}^{2} / \mathrm{s}$ (Zhao et al. 2010). The diffusion coefficient and the sample size establish a timescale for poroviscous relaxation: $\tau_{D}=10^{5} \mathrm{~s}$. When the time of observation is much shorter than the time for poroviscous relaxation, viscous relaxation is observed, and is independent of the sample size. Viscous relaxation is attributed to the continuous breaking of the ionic crosslinks. The timescale depends on the calcium concentration and the distance between crosslinks. The viscous relaxation time is of the order of 
10s (Mitchell 1980, Zhao et al. 2010, Gentile et al. 2013). When the time of observation is much larger than the time for viscous relaxation, Gentile et al. (2013) reported a transition to a diffusion-dominated regime.

Another example where concurrent viscous relaxation and poroviscosity could play an important role is in cells. Cells broadly consist of the cytoplasm - a gel-like solution of water and macromolecules - enclosed in a water permeable membrane. Water permeation permeates quickly through the membrane, but much slower through the cytoplasm (Charras et al. 2009). Recently, the poroelastic theory was successfully applied to describe various aspects of the cell response (Charras et al., 2005, 2009). A typical cell as a size of the order of $10 \mu \mathrm{m}$. The diffusivity of water in the cell is $D=10^{-11} \mathrm{~m}^{-2} / \mathrm{s}$ (Charras et al. 2005), which gives a timescale for poroelastic relaxation is $\tau_{D}=10 \mathrm{~s}$. This timescale is relevant for biological processes, which take places over the timescales of the order 10-100s, such as cell migration or blebbing. However, experiments using AFM showed that the timescale for viscous relaxation is also of the order of 10s, while in this case poroelasticity has relaxed (Darling et al. 2007). Therefore, it is conceivable that both poroelasticity and viscosity act concurrently.

To focus on viscous mixing, we have neglected elasticity and assumed infinitely fast diffusion. As such, the theory is applicable to viscous fluids of small size compared to the poroviscous length. However, the idea of viscous mixing can be included within a more comprehensive theory that includes elasticity and diffusion to address a broader class of problems coupling interdiffusion and mechanics. Our formulation will be directly relevant to address problems in amorphous system such as glasses, polymers and gels where inelastic deformations and species accommodation are mediated by similar molecular processes.

\section{ACKNOWLEDGMENTS}

This work at Harvard is supported by MRSEC (DMR 14-20570). L. Brassart acknowledges the support of the National Fund for Research of Belgium (FNRS). 


\section{References}

Angell, C. A., Ngai, K. L., McKenna, G. B., McMillan, P. F., Martin, S. W., 200o. Relaxation in glassforming liquids and amorphous solids. Applied Physics Review 88, 3113-3157.

Berthier, L., 2011. Dynamic heterogeneity in amorphous materials. Physics 4, 42.

A.F. Bower, P.R. Guduru, V.A. Sethuraman, 2011. A finite strain model of stress, diffusion, plastic flow, and elecrochemical reactions in lithium-ion half-cell. Journal of the Mechanics and Physics of Solids 59, 804-828.

Bargmann, S., McBride, A.T., Steinmann, P., 2011. Models of solvent penetration in glassy polymers with an emphasis on case II diffusion. A comparative review. Applied Mechanics Reviews 64, 010803.

Brassart, L., Suo, Z., 2012. Reactive flow in large-deformation electrodes of lithium-ion batteries. International Journal of Applied Mechanics 4, 1250023.

Brassart, L., Suo, Z., 2013. Reactive flow in solids. Journal of the Mechanics and Physics of Solids 61, 61-77.

Brassart, L., Zhao, K., Suo, Z., 2013. Cyclic plasticity and shakedown in high-capacity electrodes of lithium-ion batteries. International Journal of Solids and Structures 50, 1120-1129.

Chester, S.A., 2012. A constitutive model for coupled fluid permeation and large viscoelastic deformation in polymeric gels. Soft Matter 8, 8223-8233.

Coble, R. L., 1963. A Model for Boundary Diffusion Controlled Creep in Polycrystalline Materials. Journal of Applied Physics 34, 1679-1682.

Cui, Z., Gao, F., Qu, J., 2012. A finite deformation stress-dependent chemical potential and its application to lithium-ion batteries. Journal of the Mechanics and Physics of Solids 60, 1280-1295.

Darken, L.S., 1948. Diffusion, mobility and their interrelation through free energy in binary metallic systems. Transactions of the American Institute of Mining and Metallurgical Engineers 175, 184-201. 
Debenedetti, P.G., Stillinger, F. H., 2001. Supercooled liquids and the glass transition. Nature 410, 259-267.

De Groot, S.R., Mazur, P., 1984. Non-equilibrium thermodynamics, $2^{\text {nd }}$ Edition. North-Holland.

Di Leo, C. V., Rejovitzky, E., Anand, L., 2015. Diffusion-deformation theory for amorphous silicon anodes: The role of plastic deformation on electrochemical performance. International Journal of Solids and Structures 67-68, 283-296.

Drozdov, A.D., 2014. Viscoplastic response of electrode particles in Li-ion batteries driven by insertion of lithium. International Journal of Solids and Structures 51, 690-705.

Durning, C.J., 1985. Differential sorption in viscoelastic fluids. Journal of Polymer Science 23, 1831-1855.

Ediger, M.D., Harrowell, P., 2012. Perspective: supercooled liquids and glasses. The Journal of Chemical Physics 137, 080901.

Edward, J. T., 1970. Molecular volumes and the Stokes-Einstein relation. Journal of Chemical Education 47, 261-270.

Einstein, A., 1905. Über die von der molekularkinetischen Theorie der Wärme geforderte Bewegung von in ruhenden Flüssigkeiten suspendierten Teilchen. Annalen der Physik 322, 549-560.

Faupel, F., Werner, F., Macht, M.-P., Mehrer, H., Naundorf, V., Rätzke, K., Schober, H. R., Sharma, S. K., Teichler, H., 2003. Diffusion in metallic glasses and supercooled melts. Reviews of Modern Physics 75, 237-280.

Flory, P.J., 1942. Thermodynamics of high polymer solutions. Journal of Chemical Physics 10, 51-61.

Frost H. J., Ashby, M. F., 1982. Deformation-mechanism maps: the plasticity and creep of metals and ceramics. Pergamon Press.

Govindjee, S., Simo, J.C., 1993. Coupled stress-diffusion: case II. Journal of the Mechanics and Physics of Solids 41, 863-887.

Herring, C., 1950. Diffusional Viscosity of a Polycrystalline Solid, Journal of Applied Physics 21, 
437-445.

Hu, Y., Suo, Z., 2012. Viscoelasticity and poroelasticity in elastomeric gels. Acta Mechanica Solida Sinica 25, 1-18.

Huggins, M. L., 1941. Solutions of long chain compounds. The Journal of Chemical Physics 9, 440.

Landau, L.D., Lifshitz, E.M., 1987. Fluid mechanics, $2^{\text {nd }}$ Edition. Butterworth-Heinemann.

Li, J., Liu, Q., Brassart, L., Suo, Z., 2014. Mechanics of supercooled liquids. Journal of Applied Mechanics 81, 111007.

Liu, Q., Huang, S., Suo, Z., 2015. Brownian motion of molecular probes in supercooled liquids. Physical Review Letters 114, 224301.

Loeffel, K., Anand, L., 2011. A chemo-thermo-mechanically-coupled theory for elasticviscoplastic deformation, diffusion, and volumetric swelling due to a chemical reaction. International Journal of Plasticity 27, 1409-1431.

McKenzie, D., 1984. The generation of compaction of partially molten rock. Journal of Petrology $25,713-765$.

Mishin, Y., Warren, J.A., Sekerka, R.F., Boettinger, W.J., 2013. Irreversible thermodynamics of creep in polycrystalline solids. Physical Review B 88, 184303.

Nabarro, F. R. N., 1948. Deformation of Crystals by the Motion of Single Ions. Report of a Conference on Strength of Solids (Bristol), 75-90.

Needleman, A., Rice, J. R., 1980. Plastic creep flow effects in the diffusive cavitation of grain boundaries. Acta Metallurgica 28, 1315-1332.

Pharr, M., Zhao, K., Suo, Z., Ouyang, F.-Y., Liu, P., 2011. Concurrent electromigration and creep in lead-free solder. Journal of Applied Physics 110, 083716.

Stephenson, G.B., 1988. Deformation during interdiffusion. Acta Metallurgica 36(10), 26632683.

Strange, D.G.T., Fletcher, T.L., Tonsomboon, K., Brawn, H., Zhao, X., Oyen, M.L., 2013. 
Separating poroviscoelastic deformation mechanisms in hydrogels. Applied Physics Letters 102, 031913 .

Suo, Z., Kubair, D.V., Evans, A.G., Clarke, D.R., Tolpygo, V.K., 2003. Stresses induced in alloys by selective oxidation. Acta Materialia 51, 959-974.

Suo, Z., 2004. A continuum theory that couples creep and self-diffusion. Journal of Applied Mechanics 71, 646-651.

Svoboda, J., Fischer, F.D., Fratzl, P., 2006. Diffusion and creep in multi-component alloys with non-ideal sources and sinks for vacancies. Acta Materialia 54, 3043-3053.

Thomas, N.L., Windle, A.H., 1982. A theory of case II diffusion. Polymer 23, 529-542.

Viesca, R.C., Templeton, E.L., Rice, J.R., 2008. Off-fault plasticity and earthquake rupture dynamics: 2. Effects of fluid saturation. Journal of Geophysical Research 113, Bo9307.

Wang, X., Hong, W., 2012. A visco-poroelastic theory for polymeric gels. Proceedings of the Royal Society A 468, 3824-3841.

Zhao, X., Huebsch, N., Mooney, D.J., Suo, Z., 2010. Stress-relaxation behavior in gels with ionic and covalent crosslinks. Journal of Applied Physics 107, 063509. 